\title{
OPEN Relationship between serum total testosterone and prostate volume in aging men
}

\author{
Bo-Wen Xia, Si-Cong Zhao, Zong-Ping Chen, Chao Chen, Tian-Shu Liu, Fan Yang \& Yong Yan ${ }^{\bowtie}$
}

Total testosterone levels decline with age, while prostate volume and the prevalence of benign prostatic hyperplasia increase with age. We sought to investigate the correlation of serum testosterone levels with prostate volume in aging men. We analyzed clinical data obtained from 416 ostensibly healthy men who underwent routine health check-ups and recruited and collected data from these subjects 4 years later. We analyzed the correlation between prostate volume and relevant factors, as well as the correlation between changes in prostate volume and low testosterone over a 4-year period. Men with low testosterone had significantly larger prostate volume than those in the normal testosterone group $(26.86 \pm 8.75$ vs. $24.06 \pm 6.77 P=0.02)$, and subjects with low testosterone had significantly higher levels of obesity-related factors, including waist circumference, body mass index, and insulin (all $P<0.001$ ). After adjustment for age, testosterone level was negatively correlated with prostate volume $(P=0.004)$, and prostate volume and 4 -year changes in prostate volume were associated with low testosterone. With increased testosterone level, prostate volume showed a significant linear decreasing trend. These findings provide evidence of the relationship between testosterone and prostate volume. Additional large studies are needed to confirm these preliminary results.

Benign prostate hyperplasia $(\mathrm{BPH})$ is the most prevalent benign disease and main cause of lower urinary tract symptoms (LUTS) in aging men. BPH affects the quality of life of men by gradually leading to prostate volume (PV) enlargement and resulting in LUTS, recurrent urinary tract infections, acute urinary retention and other clinical symptoms ${ }^{1}$. Age is considered to be the most important risk factor for BPH, and the clinical symptom progression of $\mathrm{BPH}$ is also closely related to the increase in PV caused by advanced age $\mathrm{e}^{2}$. Because of the high prevalence of BPH/LUTS in aging men and the high cost of treatment and early identification of patients at risk for progression events, it is essential to further identify risk factors for BPH/LUTS morbidity and progression ${ }^{3}$.

Studies have shown that testosterone is important for prostate growth and maintenance of functional integrity ${ }^{4}$. In recent years, androgen deficiency in aging men, later renamed late-onset hypogonadism, has become a frequent topic of discussion among andrologists, dermatologists, endocrinologists, and urologists ${ }^{5}$. Testosterone has important effects on cardiovascular and liver disease as well as mental health, and androgen deficiency may adversely affect organ system function and lead to severe quality of life impairment ${ }^{6,7}$. The incidence of testosterone deficiency is estimated at approximately $20 \%$ among men over the age of $60,30 \%$ over 70 and $50 \%$ over $80^{8}$. A study suggested that, independent of multiple risk factors and several preexisting health conditions, low levels of circulating total testosterone are associated with a $40 \%$ increase in mortality in older men .

Although testosterone is closely related to PV, both change significantly with age, but how testosterone affects PV is unclear. Partly based on human cross-sectional ${ }^{10-12,14,15,33-34}$ and longitudinal studies ${ }^{13,16}$ researchers have found that elevated testosterone levels increase PV, while others have found no or negative correlation between prostate volume and testosterone. Thus, in older men, the effects of androgens on PV and LUTS remain controversial. Current studies have found that androgen replacement therapy (TRT) can relieve LUTS symptoms ${ }^{17}$, and high serum testosterone levels are associated with reduced risk of $\mathrm{BPH}^{18}$. Therefore, it has been suggested that appropriate testosterone may reduce the inflammatory response in the prostate ${ }^{19}$.

Some research has yielded results, but more systematic studies are needed to clarify the relationship between testosterone and PV in aging men. In addition, few studies have looked at the correlation between androgen and $\mathrm{PV}$ in Asian populations, and there is also an effect of race on androgen levels ${ }^{13,14}$. Therefore, in the present study, we aimed to explore the relationship between serum testosterone levels and several related factors, especially PV, in aging Asian men by collecting data from healthy Chinese people. 


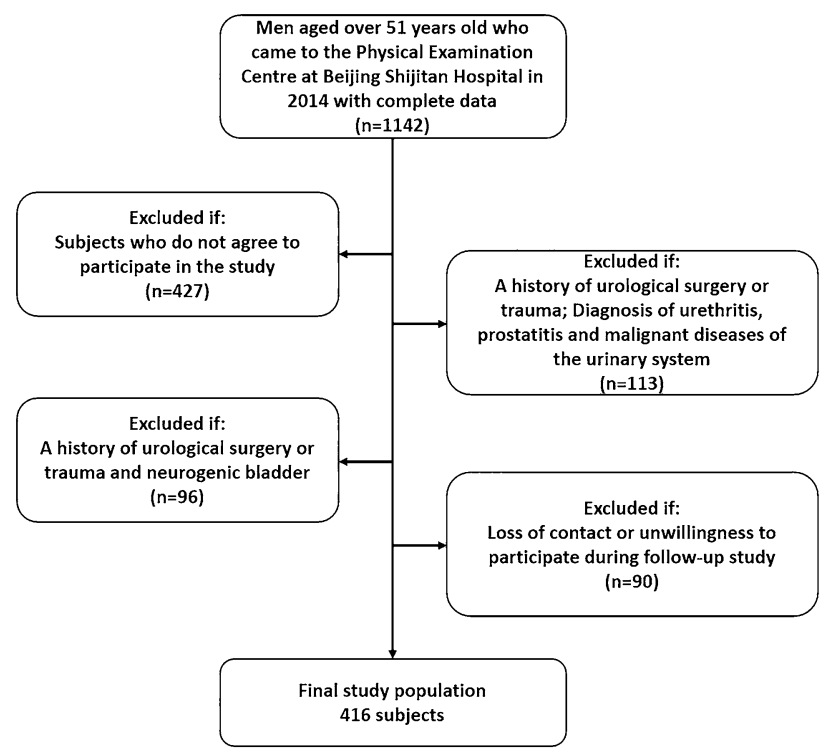

Figure 1. Flow chart of selection of the included subjects.

\begin{abstract}
Methods
Study subjects. All subjects were recruited consecutively by routine physical examination. After excluding those who met the exclusion criteria and who did not agree to participate in our research, 416 subjects aged over 51 years in the Beijing area participated in the present study by two routine physical examination programs from October 2014 and October 2018 (Fig. 1). Only men who completed the follow-up were included in the analysis. Our research used the following exclusion criteria for both data collection periods: (1) urethritis and prostatitis, urethral stricture, or neurogenic bladder; (2) a history of urological surgery or trauma; (3) diagnosis of malignant diseases of the urinary system; and (4) neurological diseases that may affect urinary function. These study protocols were approved by the ethics committee of Beijing Shijitan Hospital affiliated with Capital Medical University and China Railway Corporation, and all subjects volunteered to participate in the experiment after providing informed consent and agreeing in writing prior to registration. All experiments were performed in accordance with relevant guidelines and regulations.
\end{abstract}

Data collection. All subjects (including those enrolled in October 2014 and October 2018) completed the International Prostate Symptom Sore (I-PSS) questionnaire after Chinese translation ${ }^{20}$, and anthropometric measurements, including waist circumference $(\mathrm{cm})$, blood pressure $(\mathrm{mmHg})$, body weight $(\mathrm{kg})$ and height $(\mathrm{cm})$, were measured by trained nurses using a standardized protocol. Body mass index (BMI) was calculated by using the formula (weight/height squared), and $\mathrm{PV}(\mathrm{cm} 3)$ was measured by suprapubic ultrasonography $(3.5 \mathrm{MHz}$; Hitachi EUB-400, Tokyo, Japan) using the formula for an elliptic volume (height $\times$ width $\times$ length $\times \pi / 6$ ); the maximum urinary flow rate (Qmax) was determined by uroflowmetry at a voided volume of $>150 \mathrm{ml}$.

All blood measurements were performed using fresh serum obtained after a 12 -h fast to minimize the confounding effects of diurnal variation on hormone concentrations. The measured parameters included prostatespecific antigen (PSA), fasting blood glucose (FBG), triglycerides, high-density lipoprotein cholesterol (HDL-C), and low-density lipoprotein cholesterol (LDL-C). Insulin, $5 a$-dihydrotestosterone (DHT), sex hormone-binding globulin (SHBG), total testosterone, leptin, resistin, adiponectin, IL-6, C-reactive protein (CRP) and TNF- $\alpha$ were measured using enzyme-linked immunosorbent assay methods. The homeostasis model assessment of insulin resistance (IR) index was calculated using the HOMA algorithm: glucose $(\mathrm{mg} / \mathrm{dL}) \times$ insulin $(\mu \mathrm{U} / \mathrm{mL}) / 405^{21}$. Blood biochemical tests were collected only from the 416 subjects recruited from October 2014 to August 2015 and were tested in a single batch to reduce variability.

Statistical analysis. We defined a low testosterone level as $<300 \mathrm{ng} / \mathrm{dL}$. Clinical characteristics were compared between subjects with and without low testosterone comparatively by using independent t-tests for continuous variables and chi-square tests for categorical variables. The Shapiro-Smirnov test was used to verify whether the data fit a normal distribution, all continuous variables that did not conform to the normal distribution were analyzed according to the distribution, and all non-normal variables conformed to the normalized data after transformation. Age-adjusted linear regression models were used to evaluate the associations of related factors with PV values. Logistic regression models adjusted for only age or age and insulin were used to assess associations of low testosterone and PV levels and to calculate odds ratios (ORs). Furthermore, we divided subjects into four groups based on the quartiles of serum levels of testosterone to investigate whether there was a linear difference in PV values among the groups by using ANOVA. The data were analyzed using SPSS software version 13.0 for Windows (SPSS Inc., Chicago, IL, USA), and two-tailed $P$ values $<0.05$ indicated statistical significance. 


\begin{tabular}{|c|c|c|c|c|}
\hline Characteristics & Overall (416) & Low Testosterone(61) & Normal Testosterone(355) & $P$-value \\
\hline Age & $68.99 \pm 8.18$ & $69.15 \pm 7.99$ & $68.00 \pm 9.20$ & 0.359 \\
\hline BMI, kg/m2 & $24.76 \pm 3.13$ & $26.40 \pm 3.34$ & $24.47 \pm 3.01$ & $<0.001$ \\
\hline Waist circumference, $\mathrm{cm}$ & $86.80 \pm 8.69$ & $91.75 \pm 8.87$ & $85.95 \pm 8.38$ & $<0.001$ \\
\hline SBP, mmHg & $137.13 \pm 15.66$ & $138.39 \pm 15.47$ & $136.91 \pm 15.71$ & 0.494 \\
\hline $\mathrm{DBP}, \mathrm{mmHg}$ & $79.17 \pm 10.51$ & $79.84 \pm 11.55$ & $79.06 \pm 10.33$ & 0.593 \\
\hline Elevated blood pressure, $n(\%)$ & $70.4 \%$ & $72.1 \%$ & $70.1 \%$ & 0.879 \\
\hline FBG, $\mathrm{mg} / \mathrm{dL}$ & $102 \pm 26.01$ & $99.42 \pm 22.13$ & $103.33 \pm 26.60$ & 0.278 \\
\hline Triglycerides, mg/dL & $162.37 \pm 146.97$ & $151.64 \pm 92.35$ & $164.22 \pm 154.44$ & 0.538 \\
\hline $\mathrm{HDL}-\mathrm{C}, \mathrm{mg} / \mathrm{dL}$ & $47.31 \pm 11.24$ & $46.51 \pm 13.69$ & $47.46 \pm 10.79$ & 0.545 \\
\hline LDL-C, mg/dL & $111.42 \pm 30.52$ & $115.06 \pm 35.52$ & $110.79 \pm 29.59$ & 0.378 \\
\hline Total cholesterol, mg/dL & $187.69 \pm 37.67$ & $190.49 \pm 42.57$ & $187.21 \pm 26.80$ & 0.571 \\
\hline $\mathrm{PV}, \mathrm{cm}^{3}$ & $24.47 \pm 7.15$ & $26.86 \pm 8.75$ & $24.06 \pm 6.77$ & 0.020 \\
\hline $\mathrm{Q}_{\max }, \mathrm{mL} / \mathrm{s}$ & $15.60 \pm 6.12$ & $15.15 \pm 5.99$ & $15.68 \pm 6.14$ & 0.535 \\
\hline PSA, ng/mL & $1.39 \pm 1.16$ & $1.48 \pm 1.44$ & $1.38 \pm 1.11$ & 0.545 \\
\hline IPSS & $12.98 \pm 7.65$ & $13.36 \pm 7.86$ & $12.92 \pm 7.61$ & 0.677 \\
\hline Urination symptom score & $8.68 \pm 5.10$ & $8.95 \pm 5.24$ & $8.64 \pm 5.08$ & 0.657 \\
\hline Emptying symptom score & $4.30 \pm 2.57$ & $4.41 \pm 264$ & $4.28 \pm 2.56$ & 0.719 \\
\hline Quality of Life score & $2.87 \pm 1.60$ & $2.98 \pm 1.64$ & $2.85 \pm 1.59$ & 0.559 \\
\hline Insulin, pmol/L & $51.31 \pm 31.09$ & $66.38 \pm 38.64$ & $48.72 \pm 28.88$ & 0.001 \\
\hline HOMA IR & $2.13 \pm 1.34$ & $2.65 \pm 1.52$ & $2.04 \pm 1.29$ & 0.004 \\
\hline DHT, pg/mL & $381.84 \pm 238.25$ & $393.29 \pm 248.94$ & $315.22 \pm 147.27$ & 0.001 \\
\hline SHBG, nmol/L & $70.85 \pm 35.89$ & $46.27 \pm 27.71$ & $75.08 \pm 35.47$ & $<0.001$ \\
\hline Testosterone, ng/mL & $4.61 \pm 1.68$ & $2.56 \pm 0.48$ & $4.97 \pm 1.56$ & $<0.001$ \\
\hline
\end{tabular}

Table 1. Baseline characteristics. $P$ were calculated by independent t-test $\dagger$ and chi-squared test $\ddagger$. Bold indicates statistically significant values. The total number of subjects was 416 . BMI body mass index, $S B P$ systolic blood pressure, $D B P$ diastolic blood pressure, $F B G$ fasting blood glucose, $H D L-C$ high-density lipoprotein cholesterol, $L D L-C$ low-density lipoprotein cholesterol, $P V$ prostate volume, $Q \max$ the maximum urinary flow rate, $P S A$ prostate-specific antigen, IPSS International Prostate Symptom Sore, $D H T$ $5 a$-dihydrotestosterone, $S H B G$ sex hormone binding globulin, $F A I \%$ free androgen index.

\section{Results}

The baseline characteristics of the subjects are presented in Table 1 . The mean age in the study sample was $69.3 \pm 8.31$ years, and the mean TT level was $4.61 \pm 1.68 \mathrm{ng} / \mathrm{ml}$. The mean IPSS of the study population was 12.98 of 35, and the mean PV was $24.47 \mathrm{~cm} 3$. BMI, waist circumference, PV, MetS, PV, insulin, HOMA IR, DHT, and SHBG, levels were significantly different between subjects in the low testosterone and normal testosterone groups (all $P<0.05)$. In addition, subjects in the lower TT group were significantly more obese (BMI: $26.40 \pm 3.34$ vs. $24.47 \pm 3.01 P<0.001$; waist circumference: $91.75 \pm 8.87$ vs. $85.95 \pm 8.38 P<0.001)$ and had larger $P V(26.86 \pm 8.75$ vs. $24.06 \pm 6.77 P=0.02$ ) than those in the control group.

The results of the age-adjusted linear regression models shown in Table 2 indicated that BMI, waist circumference, blood pressure (including systolic pressure and diastolic pressure), insulin, HOMA IR, SHBG and TT were significantly correlated with PV levels (all $P<0.05$ ). Among the indicators related to obesity, BMI, waist circumference, insulin and IR were positively correlated with PV levels, while serum TT levels and SHBG were negatively associated with PV levels $(P=0.004$ and $P=0.042$, respectively). There was no significant correlation between DHT, estradiol and PV levels.

As shown in Table 3, there was a significant association of PV with low testosterone after adjusting for age $(\mathrm{OR}=1.052 \mathrm{P}=0.004)$. Low testosterone was also correlated with $\mathrm{PV}$ values after 4 years of follow-up $(\mathrm{OR}=1.056$ $P=0.002)$, and the PV growth of the low testosterone group was significantly greater than that of the normal testosterone group after 4 years $(\mathrm{OR}=3.108 \mathrm{P}<0.001)$. However, after adjusting for age, $\mathrm{BMI}$, and insulin, only the PV value difference over the 4-year period was significantly associated with low testosterone $(\mathrm{OR}=2.642$ $P<0.001)$.

Finally, we divided subjects into four groups based on the quartile serum testosterone levels to investigate whether there was a trend deviation of PV levels among the groups (Fig. 2). After adjusting for age, the mean $\mathrm{PV}$ and PV after 4 years significantly and gradually decreased as serum testosterone levels increased $(P=0.003$ and $P=0.002$, respectively). 


\begin{tabular}{|l|l|l|l|c|}
\hline Characteristics & \multicolumn{1}{l}{ B } & $\boldsymbol{t}$ & $\mathbf{9 5 \%}$ CI & $\dagger$-value \\
\hline BMI, kg/m2 & 0.322 & 6.620 & $0.226,0.417$ & $<\mathbf{0 . 0 0 1}$ \\
\hline Waist circumference, cm & 0.312 & 6.610 & $0.222,0.411$ & $<\mathbf{0 . 0 0 1}$ \\
\hline SBP, mmHg & 0.215 & 4.278 & $0.116,0.314$ & $<\mathbf{0 . 0 0 1}$ \\
\hline DBP, mmHg & 0.120 & 2.399 & $0.022,0.218$ & $\mathbf{0 . 0 1 7}$ \\
\hline FBG, mg/dL & -0.017 & -0.349 & $-0.114,0.079$ & 0.727 \\
\hline Triglycerides, mg/dL & 0.068 & 1.382 & $-0.029,0.166$ & 0.168 \\
\hline HDL-C, mg/dL & -0.063 & -1.287 & $-0.160,0.033$ & 0.199 \\
\hline LDL-C, mg/dL & -0.039 & -0.793 & $-0.135,0.057$ & 0.428 \\
\hline Total cholesterol, mg/dL & -0.077 & -1.578 & $-0.173,0.019$ & 0.115 \\
\hline Insulin, pmol/L & 0.155 & 3.102 & $0.057,0.253$ & $\mathbf{0 . 0 0 2}$ \\
\hline HOMA IR & 0.140 & 2.839 & $0.043,0.237$ & $\mathbf{0 . 0 0 5}$ \\
\hline DHT, pg/mL & -0.056 & -1.137 & $-0.153,0.041$ & 0.256 \\
\hline SHBG, nmol/L & -0.107 & -2.044 & $-0.209,-0.004$ & $\mathbf{0 . 0 4 2}$ \\
\hline Testosterone, ng/mL & -0.141 & -2.886 & $-0.237,-0.045$ & $\mathbf{0 . 0 0 4}$ \\
\hline FAI \% & -0.004 & -0.070 & $-0.106,0.099$ & 0.944 \\
\hline
\end{tabular}

Table 2. Age-adjusted regression analysis of factors that affect PV levels. Bold indicates statistically significant values. $C I$ confidence interval, $P V$ prostate volume, $B M I$ body mass index, $S B P$ systolic blood pressure, $D B P$ diastolic blood pressure, $F B G$ fasting blood glucose, $H D L-C$ high-density lipoprotein cholesterol, $L D L-C$ lowdensity lipoprotein cholesterol, DHT 5a-dihydrotestosterone, SHBG sex hormone binding globulin, FAI\%, free androgen index. ${ }^{\dagger} P$-value were calculated according to multivariate linear regression analyses after adjustment of age.

\begin{tabular}{|l|l|l|r|}
\hline Characteristics & OR & 95\% CI & P-value \\
\hline Adjustment for Age & 1.052 & $1.016,1.090$ & $\mathbf{0 . 0 0 4}$ \\
\hline PV cm & 1.056 & $1.021,1.093$ & $\mathbf{0 . 0 0 2}$ \\
\hline PV-4 year after $\mathrm{cm}^{3}$ & 3.108 & $1.838,5.255$ & $<\mathbf{0 . 0 0 1}$ \\
\hline *PV value difference cm ${ }^{3}$ & \multicolumn{5}{|l|}{} \\
\hline Adjustment for Age, BMI, Insulin \\
\hline PV cm & 1.027 & $0.990,1.066$ & 0.152 \\
\hline PV-4 year after $\mathrm{cm}^{3}$ & 1.031 & $1.000,1.017$ & 0.096 \\
\hline${ }^{*} P V$ value difference $\mathrm{cm}^{3}$ & 2.642 & $1.539,4.536$ & $<\mathbf{0 . 0 0 1}$ \\
\hline
\end{tabular}

Table 3. Associations of Low testosterone and PV levels after 4 years. ${ }^{\star} \mathrm{PV}$ value difference, $\mathrm{PV}-4$ year after subtract PV.

\section{Discussion}

The association between total testosterone levels and prostate volume has been controversial. The results of the present study show that TT level was negatively correlated with PV after adjusting for age in Chinese aging men. Meanwhile, the rate of PV growth in aging patients with low testosterone was significantly greater than the normal TT level group after adjusting for age.

As an increasing number of countries have aging societies, scientists are concerned about symptoms in older men associated with changes in sex hormones, particularly changes in testosterone levels ${ }^{22}$. Testosterone levels decrease with age, a syndrome defined by the ISSAM (International Society for the Study of the Aging Male) as androgen deficiency or late-onset hypogonadism, which can cause a number of physical complaints, including decreased libido, erectile quality, and intellectual activity; fatigue; depression; and irritability ${ }^{23}$. An observational cross-sectional study of testosterone and age found that TT and free testosterone decreased with age $(0.4 \% /$ year and $1.2 \% /$ year $)^{24}$. Feldman, $\mathrm{H}$ A also found that serum testosterone levels declined steadily after age 40 , with an average annual decrease of $0.8 \%$ in TT. Furthermore, deteriorating health may accelerate age-related declines in testosterone levels based on longitudinal data ${ }^{22}$. Our results showed that subjects in the low androgen group were older than those in the control group, but there was no significant difference. This may have been related to the androgen grouping. In the present study, there was an association between age and TT $(P=0.002)$.

Obesity was also associated with decreased TT levels in the prospective prevalence study of Calderon, B et al. ${ }^{25}$, which determined that $45 \%$ of moderately to severely obese patients had low testosterone levels, and serum TT levels were negatively correlated with blood glucose and insulin resistance. Studies have also found that the proportion of subnormal free testosterone in obese subjects is significantly higher than that in normal 

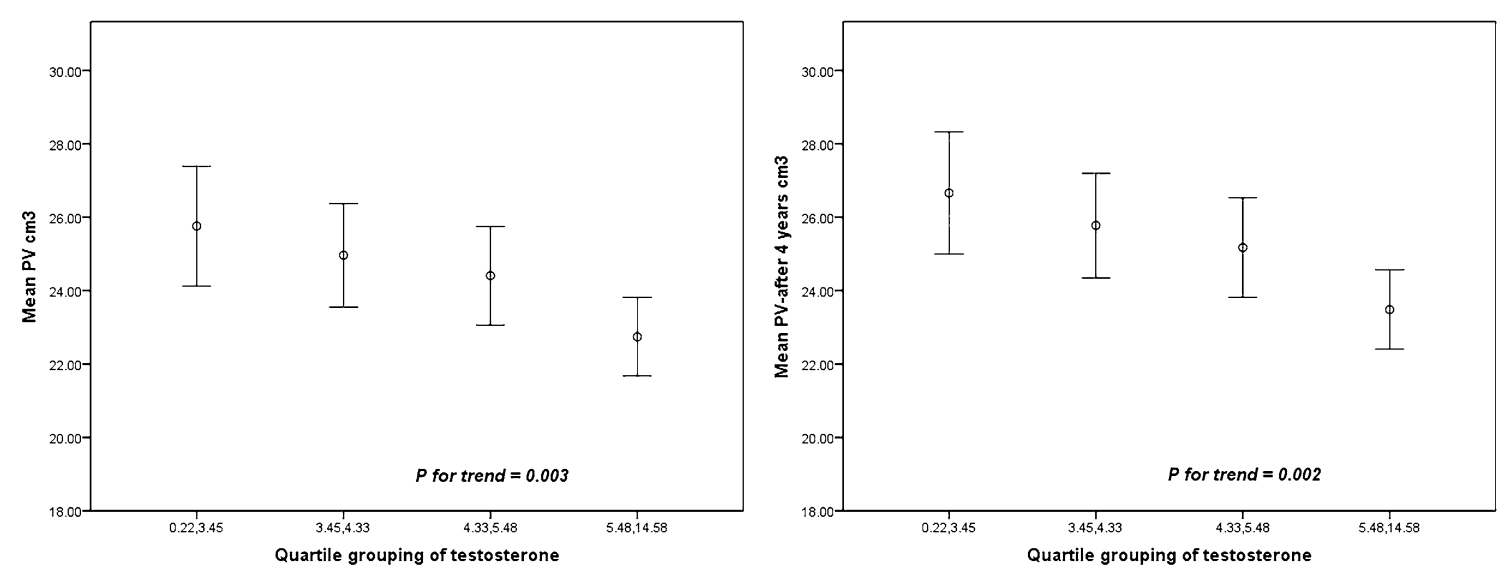

Figure 2. Mean prostate volume and prostate volume after 4 years adjusted for age. White circle indicates mean. Lower and upper bar indicates $95 \%$ CI.

and lean people, and free testosterone was negatively correlated with BMI $(r=-0.18 P<0.001)$ and significantly lower in diabetic patients. Our results also showed that subjects in the low testosterone group had significantly higher waist circumference and BMI, as well as higher insulin levels and HOMA IR, than those in the normal testosterone group.

In contrast to the relationship between TT and age, the prevalence of BPH increased significantly with age ${ }^{2}$. Some retrospective studies have also shown that there is a positive correlation between age and PV; meanwhile, the fastest increasing rate of PV was between the ages of 50 and $69^{1,26}$. In our study, we found a significant correlation between prostate volume and age $(P=0.030$, data not shown). As with TT, PV was also associated with obesity and insulin, and Vignozzi, L et al. found that obesity and insulin could have a detrimental effect on the prostate and are a risk factor for BPH progression ${ }^{27}$. A similar study indicated that PV significantly correlated with fasting serum insulin $(P=0.028)$. In our study, there was a positive correlation between PV with insulin and insulin resistance. Thus, age and obesity-related factors are both correlated with TT and PV.

Some studies have looked at the relationship between serum androgen levels in men and clinical prostatic hyperplasia or prostate volume, but the results have been inconsistent. Meikle et al. ${ }^{15}$ found that PV was negatively correlated with age-adjusted serum TT, DHT, and SHBG levels in 214 male twins between 25 and 75 years old. Another study came to a similar conclusion in Asians; with decreased TT, the IPSS score and PV all significantly increased ${ }^{10}$. Roberts et al. indicated that PV was negatively associated with bioavailable testosterone level $(\mathrm{rs}=-0.13, P<0.05)$. However, after adjusting for age, the results were not statistically significant ${ }^{13}$. In contrast, Nukui $\mathrm{M}$ et al. ${ }^{35}$ found that PV was positively correlated with TT, but this finding only existed in groups with $\mathrm{PV}>25 \mathrm{ml}$ in a cross-sectional study of 226 subjects. Other studies have also failed to find a significant association between TT level and PV ${ }^{12,14}$.

In fact, both TT and PV are correlated with race and age, and the heterogeneity of different studies may arise from the differences in age and region of the subjects. Our present results suggest that there is a significant negative association between PV and TT levels after age adjustment, and subjects with low testosterone levels had significantly larger prostate volumes than men with normal testosterone levels after 4 years. After adjusting for age, BMI and insulin levels, there was no statistical association between PV and TT levels; however, there was still a statistical correlation between increased PV over 4 years and TT levels $(O R=2.642 P<0.001)$. Similar to our current findings, a cross-sectional study of 406 Australians found a negative correlation between age and serum testosterone levels $(\mathrm{r}=-0.265 ; P<0.001)$, and BMI was inversely correlated to TT $(\mathrm{r}=-0.42 ; P<0.001)$. After adjusting for age and sampling time, PV measured by transrectal ultrasound was negatively correlated with TT, free testosterone, and bioavailable testosterone ${ }^{36}$.

TRT is increasingly used in older men. A study of 13 hypogonadal men aged 25 to 35 who underwent TRT found a significant increase in PV $(P<0.001)^{27}$. Other longitudinal researchers have shown that testosterone supplementation increases PV by an average of $12 \%{ }^{29}$. However, Morales ${ }^{30}$ found that there was no significant difference in PV between men treated with testosterone and those treated with placebo. A well-controlled RCT study of 44 men with hypogonadism showed no significant increase in testosterone levels in prostate tissue and little change in treatment-related prostate volume in men treated with TRT, despite significantly increased serum TT levels ${ }^{31}$. Long-term testosterone therapy in hypogonadal men showed significant improvements in urinary function and QoL, and PV was also correlated with testosterone treated ${ }^{32}$. In a similar study, testosterone-treated group showed a smaller increase in PV after eight years than control group ${ }^{33}$. It is possible that TRT may affect normal or low gonadal function in men with primary hypogonadism, but there is a lack of confirmation in men with aging hypogonadism. In the present study, increased serum TT and PV levels showed a linear trend of significant decrease.

Among sex hormones other than TT, Schatzl et al. ${ }^{34}$ found that estradiol (but not testosterone) was associated with PV $(\mathrm{r}=0.17, P=0.01)$. Joseph et al. ${ }^{14}$ found that SHBG and endogenous steroid hormones were correlated with prostate volume, with SHBG being negatively correlated. Our study also found a negative correlation between SHBG and PV $(P=0.042)$. In older men, however, the relationship between sex steroids and PV is 
more complex than a single effect. Further systematic studies are needed to analyze the correlation between all sex hormones and PV.

This article has some shortcomings that need to be noted. Firstly, the study was implemented in a single institution setting, which may be subject to selection bias and is not fully representative of the overall population. Further scaling is needed to validate our results. Second, the current cross-sectional study cannot reach an accurate conclusion and can only provide some evidence for the follow-up study, which requires further longitudinal and prospective studies. Third, compared with mass spectrometry-based measurements, immunoassay may not be the most accurate method when serum testosterone levels are low due to the introduction of random noise into the models. Measurement errors should be reduced as much as possible by professional and skilled laboratory personnel. A further limitation was that subjects in the present study were older on average, which may have led to some other complications of aging that affect PV, such as diabetes or metabolic syndrome. The effects of these age-related diseases on the prostate are very complex and difficult to fully correct, and the mechanism by which androgen deficiency in aging men influences PV should be further studied.

\section{Conclusion}

The results of our study suggest that testosterone levels are negatively correlated with prostate volume, and BMI, waist circumference, and insulin are negatively correlated with PV in older patients. In addition, men with low testosterone developed a larger prostate than men with normal testosterone. These findings will contribute to a better understanding of the role of TT in LUTS/BPH.

Received: 14 April 2021; Accepted: 22 June 2021

Published online: 08 July 2021

\section{References}

1. McConnell, J. D. et al. The long-term effect of doxazosin, finasteride, and combination therapy on the clinical progression of benign prostatic hyperplasia. N. Engl. J. Med. 349, 2387-2398 (2003).

2. Emberton, M. et al. Benign prostatic hyperplasia: a progressive disease of aging men. Urology 61, 267-273 (2003).

3. Parsons, J. K. Modifiable risk factors for benign prostatic hyperplasia and lower urinary tract symptoms: new approaches to old problems. J. Urol. 178, 395-401 (2007).

4. Griffiths, K., Eaton, C. L., Harper, M. E., Peeling, B. \& Davies, P. Steroid hormones and the pathogenesis of benign prostatic hyperplasia. Eur. Urol. 20(Suppl 1), 68-77 (1991).

5. Lunenfeld, B. Androgen therapy in the aging male. World J. Urol. 21, 292-305 (2003).

6. Yeap, B. B. et al. Lower testosterone levels predict incident stroke and transient ischemic attack in older men. J. Clin. Endocrinol. Metab. 94, 2353-2359 (2009).

7. Beutel, M. E. et al. Correlations between hormones, physical, and affective parameters in aging urologic outpatients. Eur. Urol. 47, 749-755 (2005).

8. Harman, S. M., Metter, E. J., Tobin, J. D., Pearson, J. \& Blackman, M. R. Longitudinal effects of aging on serum total and free testosterone levels in healthy men. Baltimore longitudinal study of aging. J. Clin. Endocrinol. Metab. 86, 724-31 (2001).

9. Laughlin, G. A., Barrett-Connor, E. \& Bergstrom, J. Low serum testosterone and mortality in older men. J. Clin. Endocrinol. Metab. 93, 68-75 (2008).

10. Shim, J. S. et al. Serum testosterone levels are negatively correlated with international prostate symptom score and transitional prostate volume. Low Urin. Tract. Symptoms 10, 143-147 (2018).

11. Pejcic, T. et al. Testosterone and dihydrotestosterone levels in the transition zone correlate with prostate volume. Prostate 77, 1082-1092 (2017).

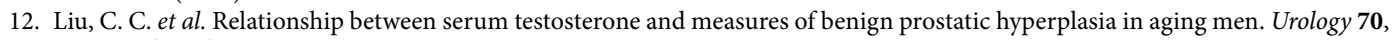
677-680 (2007).

13. Roberts, R. O. et al. Serum sex hormones and measures of benign prostatic hyperplasia. Prostate 61, 124-131 (2004).

14. Joseph, M. A. et al. Relationship of serum sex-steroid hormones and prostate volume in African American men. Prostate 53, 322-329 (2002).

15. Meikle, A. W., Stephenson, R. A., Lewis, C. M. \& Middleton, R. G. Effects of age and sex hormones on transition and peripheral zone volumes of prostate and benign prostatic hyperplasia in twins. J. Clin. Endocrinol. Metab. 82, 571-575 (1997).

16. Martin, S., Lange, K., Haren, M. T., Taylor, A. W. \& Wittert, G. Risk factors for progression or improvement of lower urinary tract symptoms in a prospective cohort of men. J. Urol. 191, 130-137 (2014).

17. Karazindiyanoglu, S. \& Cayan, S. The effect of testosterone therapy on lower urinary tract symptoms/bladder and sexual functions in men with symptomatic late-onset hypogonadism. Aging Male 11, 146-149 (2008).

18. Kristal, A. R. et al. Serum steroid and sex hormone-binding globulin concentrations and the risk of incident benign prostatic hyperplasia: results from the prostate cancer prevention trial. Am. J. Epidemiol. 168, 1416-1424 (2008).

19. Vignozzi, L. et al. Testosterone protects from metabolic syndrome-associated prostate inflammation: an experimental study in rabbit. J. Endocrinol. 212, 71-84 (2012).

20. Badia, X., Garcia-Losa, M. \& Dal-Re, R. Ten-language translation and harmonization of the International Prostate Symptom Score: developing a methodology for multinational clinical trials. Eur. Urol. 31, 129-140 (1997).

21. Matthews, D. R. et al. Homeostasis model assessment: insulin resistance and beta-cell function from fasting plasma glucose and insulin concentrations in man. Diabetologia 28, 412-419 (1985).

22. Feldman, H. A. et al. Age trends in the level of serum testosterone and other hormones in middle-aged men: longitudinal results from the Massachusetts male aging study. J. Clin. Endocrinol. Metab. 87, 589-598 (2002).

23. Morales, A. \& Lunenfeld, B. Investigation, treatment and monitoring of late-onset hypogonadism in males. Official recommendations of ISSAM. International Society for the Study of the Aging Male. Aging Male 5, 74-86 (2002).

24. Gray, A., Feldman, H. A., McKinlay, J. B. \& Longcope, C. Age, disease, and changing sex hormone levels in middle-aged men: results of the Massachusetts Male Aging Study. J. Clin. Endocrinol. Metab. 73, 1016-1025 (1991).

25. Calderon, B. et al. Prevalence of male secondary hypogonadism in moderate to severe obesity and its relationship with insulin resistance and excess body weight. Andrology-US 4, 62-67 (2016).

26. Cao, N. et al. The characteristics of the transitional zone in prostate growth with age. Urology 105, 136-140 (2017).

27. Vignozzi, L. et al. Fat boosts, while androgen receptor activation counteracts, BPH-associated prostate inflammation. Prostate 73, 789-800 (2013).

28. Sasagawa, I. et al. Volume change of the prostate and seminal vesicles in male hypogonadism after androgen replacement therapy. Int. Urol. Nephrol. 22, 279-284 (1990). 
29. Holmang, S., Marin, P., Lindstedt, G. \& Hedelin, H. Effect of long-term oral testosterone undecanoate treatment on prostate volume and serum prostate-specific antigen concentration in eugonadal middle-aged men. Prostate 23, 99-106 (1993).

30. Morales, A. Androgen replacement therapy and prostate safety. Eur. Urol. 41, 113-120 (2002).

31. Marks, L. S. et al. Effect of testosterone replacement therapy on prostate tissue in men with late-onset hypogonadism: a randomized controlled trial. JAMA 296, 2351-2361 (2006).

32. Saad, F. et al. Hypogonadal men with moderate-to-severe lower urinary tract symptoms have a more severe cardiometabolic risk profile and benefit more from testosterone therapy than men with mild lower urinary tract symptoms. Invest. Clin. Urol. 59(6), 399-409 (2018).

33. Haider, K. S. et al. Long-term testosterone therapy improves urinary and sexual function, and quality of life in men with hypogonadism: results from a propensity matched subgroup of a controlled registry study. J. Urol. 199(1), 257-265 (2018).

34. Schatzl, G. et al. Endocrine status in elderly men with lower urinary tract symptoms: correlation of age, hormonal status, and lower urinary tract function. The Prostate Study Group of the Austrian Society of Urology. Urology 55, 397-402 (2000).

35. Nukui, M., Takada, H. \& Nakagawa, S. Cross-sectional analysis between prostate volumes and serum sex hormones in Japanese men attending a urological clinic. Aging Male 19, 148-154 (2016).

36. Zhuang, T. N., Ly, L. P., Cumming, R. G. \& Handelsman, D. J. Growth and development during early manhood as determinants of prostate size in later life. J. Clin. Endocrinol. Metab. 90, 6055-6063 (2005).

\section{Acknowledgements}

This work was supported by a grant from the China Railway Corporation (No. 2017Z004-A).

\section{Author contributions:}

X.B.W., Z.S.C., Y.Y. have made substantial contributions to the designed of the work and analyzed and interpreted of the data and drafted the manuscript and revised it. C.Z.P., C.C., Y.F. and L.T.S. have made contributions to collection of the data. All authors have read and approved the final version of the manuscript, and agree with the order of presentation of the authors.

\section{Competing interests}

The authors declare no competing interests.

\section{Additional information}

Correspondence and requests for materials should be addressed to Y.Y.

Reprints and permissions information is available at www.nature.com/reprints.

Publisher's note Springer Nature remains neutral with regard to jurisdictional claims in published maps and institutional affiliations.

Open Access This article is licensed under a Creative Commons Attribution 4.0 International License, which permits use, sharing, adaptation, distribution and reproduction in any medium or format, as long as you give appropriate credit to the original author(s) and the source, provide a link to the Creative Commons licence, and indicate if changes were made. The images or other third party material in this article are included in the article's Creative Commons licence, unless indicated otherwise in a credit line to the material. If material is not included in the article's Creative Commons licence and your intended use is not permitted by statutory regulation or exceeds the permitted use, you will need to obtain permission directly from the copyright holder. To view a copy of this licence, visit http://creativecommons.org/licenses/by/4.0/.

(C) The Author(s) 2021 